\title{
A previously undescribed form of intraductal papillary neoplasm of the bile duct
}

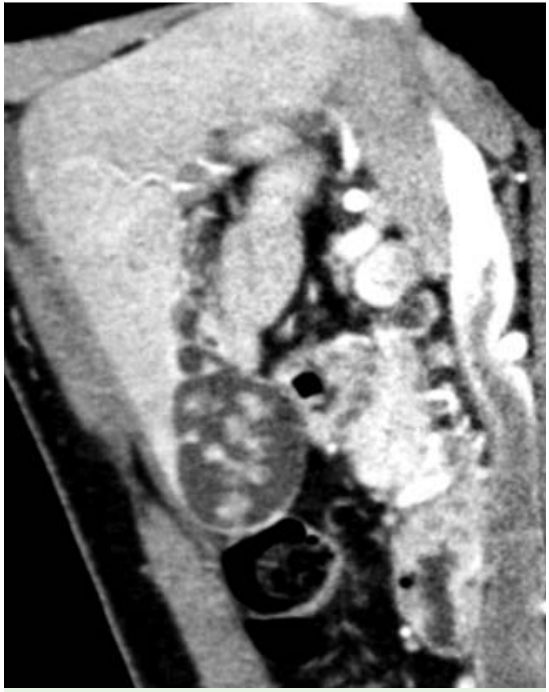

Fig. 1 Coronal view in a contrast-enhanced computed tomography (CT) scan performed on a 70-year-old man with epigastric pain showing a cystic mass with enhancing papillary tumors.

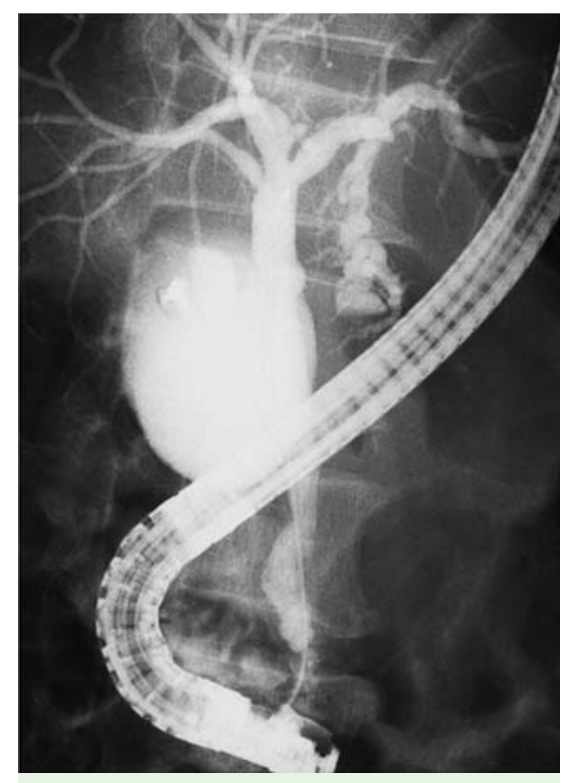

Fig.3 Image during endoscopic retrograde cholangiopancreatography (ERCP) showing that the cystic mass was in communication with the dilated intrahepatic (B4) bile duct.

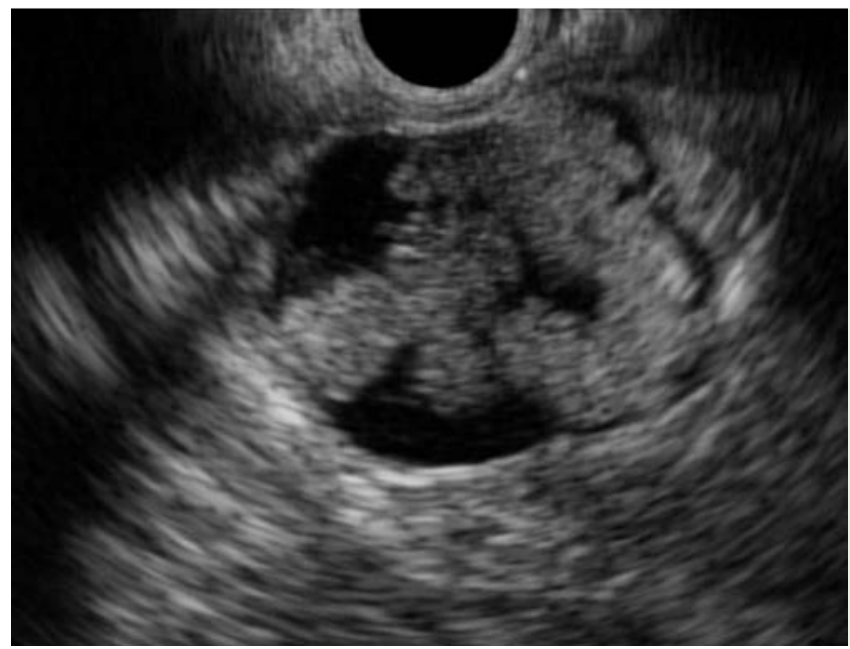

Fig. 2 Endoscopic ultrasound (EUS) image showing papillary tumors clearly visible within the cystic mass.

Intraductal papillary neoplasm of the bile duct (IPNB) is characterized by dilated intrahepatic bile ducts filled with papillary or villous neoplastic biliary epithelium [1]. Not infrequently, because of its production of excess mucin, this tumor causes cystic dilation of the affected bile ducts as well as branched-type intraductal mucinous papillary neoplasm of the pancreas [2]. Because IPNB is really located within the intrahepatic bile duct, the main site of this tumor is within the liver. Although several cases occurring in the extrahepatic duct have been reported [3], IPNB found only in an extrahepatic site that communicated with intrahepatic bile ducts has not been previously reported. We herein report the first case of an IPNB occurring in an extrahepatic site communicating with the intrahepatic bile duct.

The patient was a 70-year-old man who attended our institution with epigastric pain. All his laboratory tests were normal, including his serum levels of carbohydrate antigen 19-9 (CA19-9) and carcinoembryonic antigen (CEA). A coronal image in a contrast-enhanced computed tomography (CT) scan showed a cystic mass with enhancing papillary tumors that were connected to the intrahepatic bile duct ( Fig. 1), although the mass was extrahepatic. Therefore, we considered that the cystic mass was derived from a cystic dilated bile duct. On endoscopic ultrasound (EUS), papillary tumors in the cystic mass were clearly identified, and the outer highly echogenic layer of the wall of the cystic mass was intact ( $\bullet$ Fig. 2 ). On contrast-enhanced EUS, marked enhancement was observed from the early stage of enhancement.

We next performed an endoscopic retrograde cholangiopancreatography (ERCP), during which mucin was profusely extruded from the ampulla of Vater and the cystic mass was found to be in communication with the dilated intrahepatic (B4) bile duct ( $\bullet$ Fig.3). The filling defect in the intrahepatic bile duct was considered to be mucin. Therefore, we diagnosed this cystic mass as an IPNB and performed a surgical resection.

The findings at laparotomy were that the cystic mass was glossy and expansile but there were no invasion of other organs $(\bullet$ Fig.4). In addition, the tumor was found to originate from an extrahepatic site near the gallbladder. Histopathological examination showed a malignant papillary proliferation within the dilated B4 bile duct and invasion into the bile duct wall on hematoxylin and eosin (H\&E) staining ( $\bullet$ Fig. 5), consistent with the diagnosis of IPNB. No signs of recurrence had been observed by 1 year postoperatively.

To the best of our knowledge, the present case is the first report of an IPNB that occurred in an extrahepatic site communicating with the intrahepatic bile duct.

Endoscopy_UCTN_Code_CCL_1AZ_2AC

Competing interests: None 

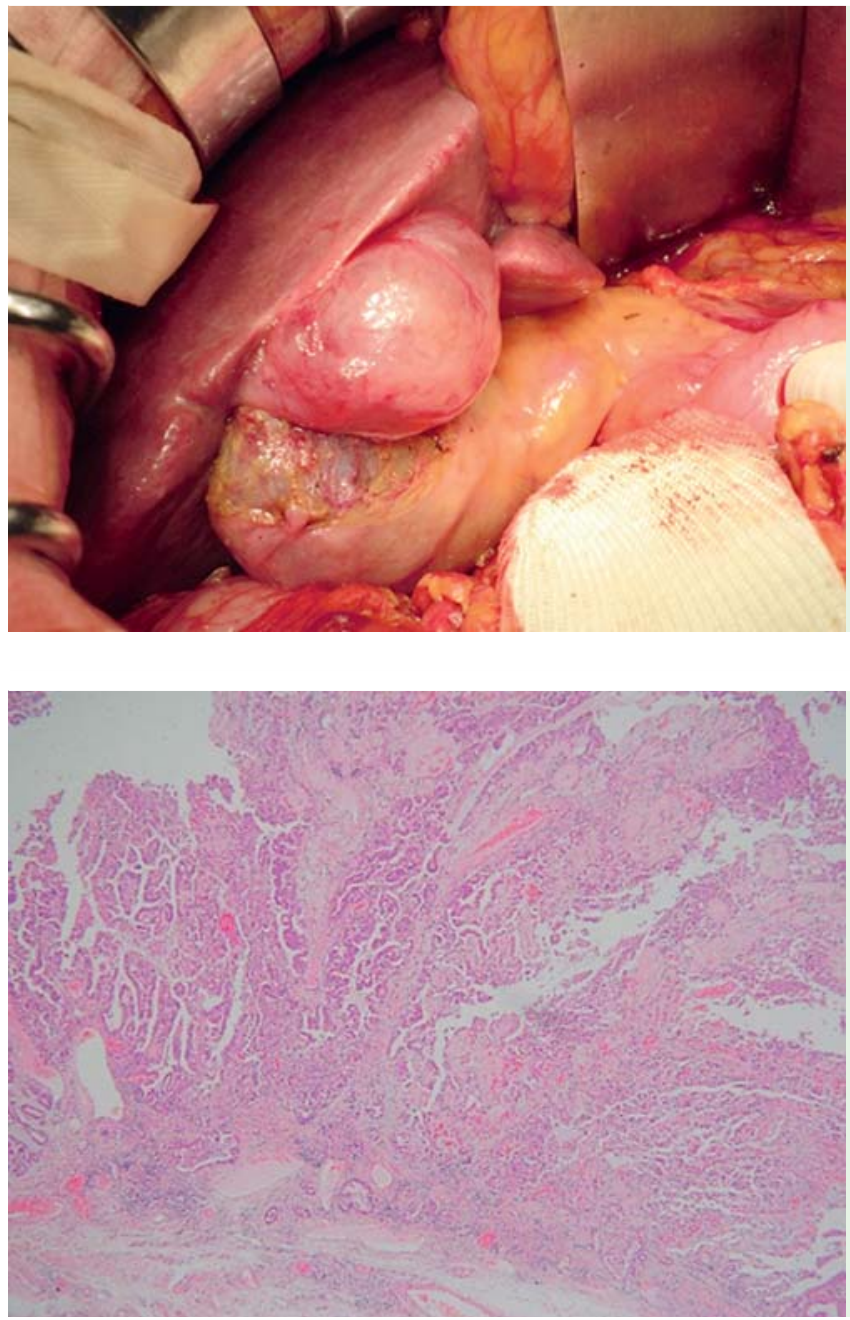

Fig.4 Appearance at laparotomy showing that the cystic mass was glossy and expansile, but there was no invasion of other organs.

T. Ogura', Y. Kurisu², D. Masuda', M. Hayashi ${ }^{3}$, A. Imoto ${ }^{1}$, E. Umegaki ${ }^{1}$, K. Uchiyama ${ }^{3}$, K. Higuchi ${ }^{1}$

1 2nd Department of Internal Medicine, Osaka Medical College, Osaka, Japan

${ }^{2}$ Department of Pathology, Osaka Medical College, Osaka, Japan

${ }^{3}$ Department of General and Gastroenterological Surgery, Osaka Medical College, Osaka, Japan

\section{References}

1 Zen $Y$, Fujii T, Itatsu $K$ et al. Biliary papillary tumors share pathological features with intraductal papillary mucinous neoplasm of the pancreas. Hepatology 2006; 44: $1333-$ 1343

2 Lim JH, Jang KT. Mucin-producing bile duct tumors: radiological-pathological correlation and diagnostic strategy. J Hepatobiliary Pancreat Sci 2010; 17: 223-229

Fig.5 Histopathological examination of the resected cystic mass with hematoxylin and eosin (H\&E) staining showing a malignant papillary proliferation within the dilated B4 bile duct.
3 Terada T. Non-invasive intraductal papillary neoplasms of the common bile duct: a clinicopathologic study of six cases. Int J Clin Exp Pathol 2012; 5: 690-697

\section{Bibliography}

DOI http://dx.doi.org/

10.1055/s-0032-1326372

Endoscopy 2013; 45: E340-E341

(C) Georg Thieme Verlag KG

Stuttgart · New York

ISSN 0013-726X

\section{Corresponding author}

\section{T. Ogura, PhD, MD}

2nd Department of Internal Medicine

Osaka Medical College

1-1 Daigakuchou, Takatsukishi,

Osaka 464-8681

Japan

Fax: +81-52-7635233

oguratakeshi0411@yahoo.co.jp 